\title{
Hand Movement Investigations Inspired by Yarbus
}

\author{
Astrid M.L. Kappers* \\ Helmholtz Institute, Utrecht University
}

\author{
Floris T. Douw \\ Helmholtz Institute, Utrecht University
}

\begin{abstract}
Inspired by the work of Yarbus [6] on eye movements, we investigated the dependence of hand movements on "scene" and question about the scene. Our haptic scenes consisted of arrays of $3 \times 3$ samples of materials that could differ in material properties or orientation of texture. Questions we asked our participants were, for example, "Describe the relief" or "Which sample is warmest?". Our results show that different questions lead to very distinctive movement patterns, such as a series of "static touches" or local "back and forth movements". Interestingly, different participants produce remarkably similar movement patterns.
\end{abstract}

Keywords: Hand movements, Exploratory movements

Index Terms: H 1.2 [Models and Principles]: User/Machine Systems-Human information processing; J.4 [Social and Behavioural Sciences]: Psychology

\section{INTRODUCTION}

In his seminal book of 1967, Yarbus [6] showed that the task given to a person, greatly influences the kind of eye movements made. If persons are asked to look at a picture and to estimate the ages of the people visible on the picture, the material circumstances of the family or how long the visitor has been away from the family, very distinct patterns of eye movement patterns are made. Yarbus, however, was not the first to investigate how people look at pictures. In 1935, Buswell [3] was probably the first to build a non-intruisive eyetracker, which he used for studying patterns of perception. These important studies mark the start of a long history of eye movement studies.

In the current study, we wondered whether something similar would occur when persons explored "haptic scenes". Would hand movements also depend on the type of question asked? From the influencing work of Lederman and Klatzky [4], we know that there is a clear link between haptic object exploration and the desired knowledge about objects. They distinguished eight so-called "exploratory procedures" that are optimal given a specific task. For example, to determine weight, observers would typically use unsupported holding, for temperature static contact and for global shape enclosure. Here we focus not on objects, but on two-dimensional haptic scenes.

Haptic exploration of two-dimensional scenes would typically occur when observers compare material properties, such as perceived temperature (heat conductance), texture, roughness, orientation of texture etc. Lederman and Klatzky [4] described the corresponding exploratory strategies as "lateral motion" for roughness and texture, and "static contact" for temperature related judgements. Another category of scannning hand movements occurs while reading Braille. These latter studies, although interesting, are not directly relevant for the current study (e.g. [2,5]).

Although lateral motion and static contact are obvious movements for exploring a two-dimensional scene, here we were in-

*e-mail: a.m.1.kappers@uu.nl terested in the variation that we would find if subjects were asked different questions about these scenes. Therefore, we constructed "scenes" by making $3 \times 3$ arrays of samples that differed in various material properties. Similar to the eye movements experiments, we asked blindfolded participants specific questions about these scenes, such as, for example, "Which of the 9 samples feels coldest?" or "Describe the relief". Questions we liked to answer are whether the movement patterns do indeed depend on the question, whether different subjects use similar movement patterns and whether different scenes lead to similar movement patterns if the same question is asked.

This paper consists of mainly a qualitative exploration of the research questions posed above. A modest start is made towards a more quantitative analysis and description of the movement patterns found.

\section{Methods}

\subsection{Set-up}

Five subjects (four male, one female) participated in this study. The subjects were blindfolded and seated behind a table on which a haptic "scene", consisting of a grid of $3 \times 3$ samples was placed (see Figure 1). Hand movements were recorded by means of a small sensor attached to the index finger of their dominant hand. An OptoTrak Certus system tracked the position of the sensor at a rate of $50 \mathrm{~Hz}$ recording hand movement patterns. The OptoTrak system yields coordinates in three dimensions, but we used only two of these as this experiment is only concerned with the perception of two-dimensional stimuli, and hence only with movements on the surface.

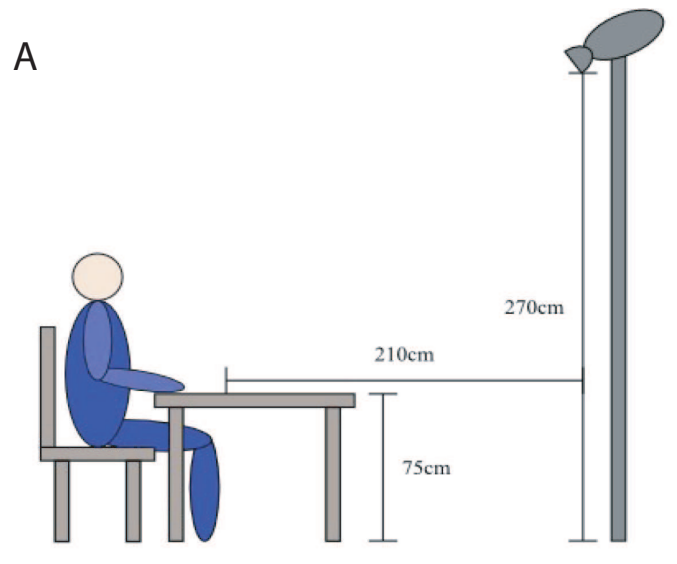

B

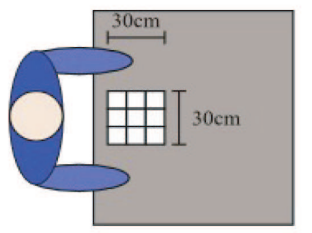

Figure 1: Schematic view of the set-up used in the experiment. A) as seen from the side, and B) top view. The object on the right is the OptoTrak tracking system. 
Table 1: Materials used in the "scenes". Materials up to number 124 come from the set of Bergmann Tiest and Kappers [1].

\begin{tabular}{ll} 
Number & Material \\
\hline 7 & Corrugated cardboard \\
8 & Synthetic felt \\
14 & Plastic carier bag \\
19 & Wrapping paper \\
48 & Insulating cardboard \\
52 & Thick teflon \\
60 & Sanding cloth Siarol P 240 \\
72 & Pressed sheeting material \\
76 & Thick steel sheeting \\
77 & Brushed aluminium sheeting \\
78 & Steel sheeting with holes \\
80 & Thin steel sheeting \\
81 & Brass sheeting \\
82 & Copper sheeting \\
83 & Ribbed aluminium block \\
84 & Bakelite sheeting \\
85 & Thin synthetic sheeting \\
92 & Thin plastic sheeting \\
93 & Nylon \\
95 & Plasterboard \\
101 & Bumpy adhesive plastic \\
102 & Wood-like adhesive plastic \\
103 & Grooved adhesive plastic \\
105 & Smooth glass \\
106 & Glass with coarse structure \\
107 & Ribbed glass \\
108 & Glass with medium structure \\
109 & Matt glass \\
110 & Glass with fine structure \\
116 & Oak veneer \\
121 & Wall tile \\
122 & Glazed floor tile \\
123 & Smooth floor tile \\
124 & Structured floor tile \\
127 & Corrugated cardboard, rotated by $45^{\circ}$ \\
132 & Corrugated cardboard, rotated by $89^{\circ}$ \\
\hline & \\
& \\
\hline &
\end{tabular}

\subsection{Stimuli}

Table 1 provides a list of the different materials used in this study. Except for the samples with numbers higher than 124, all samples have been used and described in more detail by Bergmann Tiest and Kappers [1]. The size of all samples was $10 \times 10 \mathrm{~cm}$. From these samples a number of haptic scenes were created by placing them in various combinations in a $3 \times 3$ grid. The various scenes used for illustration in the current paper are described in Figure 2. The numbers in this figure refer to the numbers in Table 1.

In addition to the materials mentioned in Table 1 , there was a series of samples consisting of corrugated cardboard with the following orientations: $0^{\circ}, 22.5^{\circ}, 45^{\circ}, 67.5^{\circ}, 89^{\circ}, 90^{\circ}, 112.5^{\circ}, 135^{\circ}$, $157.5^{\circ}$ and $179^{\circ}$. With these samples, scenes were created that consisted exclusively of corrugated cardboard in different orientations.

\subsection{Tasks}

Subjects had to explore the haptic scenes in order to be able to answer a certain question that was given to them before their exploration. In this paper we mainly focus on questions relating to temperature and relief. Each subject explored 16 scenes and the corresponding questions/tasks are given in Table 2.

Questions 1-4 focused on temperature, whereas questions 5-8 were more directly related to comparing samples to the temperature of a specific sample. Of course, the actual temperatures of the

\begin{tabular}{|c|c|c|}
\hline 93 & 107 & 85 \\
\hline 105 & 52 & 82 \\
\hline 76 & 78 & 121 \\
\hline
\end{tabular}

\begin{tabular}{|r|r|r|}
\hline 121 & 78 & 76 \\
\hline 82 & 52 & 105 \\
\hline 85 & 107 & 93 \\
\hline
\end{tabular}

\begin{tabular}{|c|c|c|}
\hline 106 & 84 & 77 \\
\hline 80 & 81 & 110 \\
\hline 108 & 123 & 122 \\
\hline
\end{tabular}

\begin{tabular}{|c|c|c|}
\hline 121 & 78 & 76 \\
\hline 82 & 52 & 105 \\
\hline 85 & 107 & 93 \\
\hline
\end{tabular}

\begin{tabular}{|c|c|c|}
\hline 116 & 19 & 101 \\
\hline 7 & 60 & 127 \\
\hline 18 & 124 & 109 \\
\hline
\end{tabular}

\begin{tabular}{|c|c|c|}
\hline 102 & 92 & 83 \\
\hline 8 & 72 & 103 \\
\hline 95 & 14 & 132 \\
\hline
\end{tabular}

Figure 2: Scenes consisting of 9 material samples as used in the various tasks that are illustrated in the examples in this paper. Upper row: scenes 1, 3, 6; bottom row: scenes $7,9,10$. The numbers in the scenes refer to the materials in Table 1.

Table 2: Questions/tasks given to subjects when they had to explore a scene with the corresponding number.

\begin{tabular}{ll} 
Number & Question/task \\
\hline $1-2$ & Which sample feels coldest? \\
$3-4$ & Which sample feels warmest? \\
5 & Which sample feels most like the top-right in terms \\
& of temperature? \\
6 & Which sample feels most like the left-center? \\
$7-8$ & Which sample feels most like the top-center? \\
$9-10$ & Describe the relief. \\
$11-12$ & Describe the direction of the ridges in the material. \\
$13-16$ & On which material are the ridges oriented most \\
& like those on the top-right?
\end{tabular}

samples did not differ as they were all at room temperature, but due to different physical properties such as heat conductance and heat capacity, the perceived temperature could vary. Questions 9-12 were related to relief or orientation of relief, although on purpose not all samples had a distinct relief or orientation. Questions 13-16 were also directed towards orientation of relief. In the corresponding scenes, the samples consisted of corrugated cardboard of which the orientation of the ridges varied systematically.

Movement recordings were made during the whole period of exploration. As we were only interested in the movement patterns in relation to the question, we did not score whether the answer the subject gave was correct.

\section{Results}

In Figure 3 examples of movement patterns are shown when the task of the subject was to find the coldest (upper row) or warmest (bottom row) sample. Note that what is plotted is the position of the sensor on the index finger. In general, subjects used their whole hand, so even samples that seem not to be touched at all, are touched by a different part of the finger or hand. Interestingly, subjects hardly visit a sample again, so apparently this task is easy. Although the local velocities cannot be seen in plots like these, it is clear that subjects make systematic scanning movements from one sample to the next, without making many detailed movements within a sample. As the questions were related to temperature, this suggests that subjects were using "static touch", the exploratory movement identified by Lederman and Klatzky [4] for situations like these. After the experiment, subject 5 indicated that he had somewhat misunderstood the instructions: he had thought that he was not supposed 
1.1

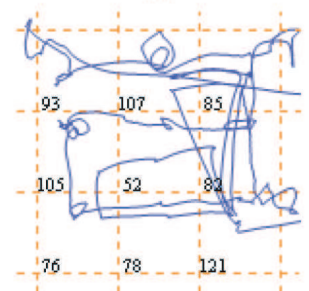

13



2.1

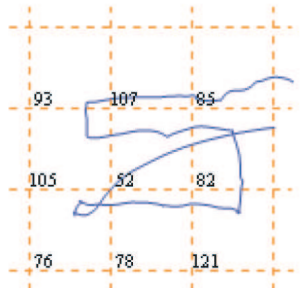

2.3



3.1

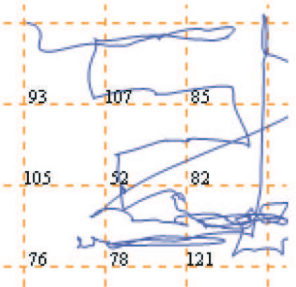

3.3

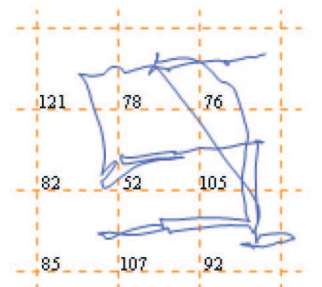

4.1



43

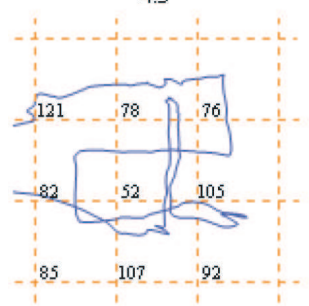

5.1

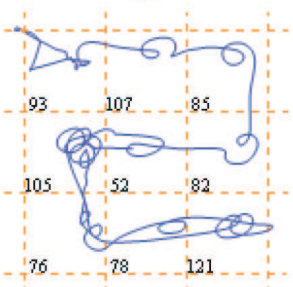

5.3

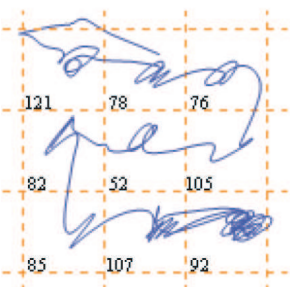

Figure 3: Movements patterns for five subjects resulting from the questions "Which sample feels coldest?" (upper row) or "Which sample feels warmest?" (bottom row). Each item represents the hand movement pattern on an array of $3 \times 3$ material samples. The first number above each scene refers to the subject, the second to the scene number. Scenes are described in Table 1 and Figure 2.

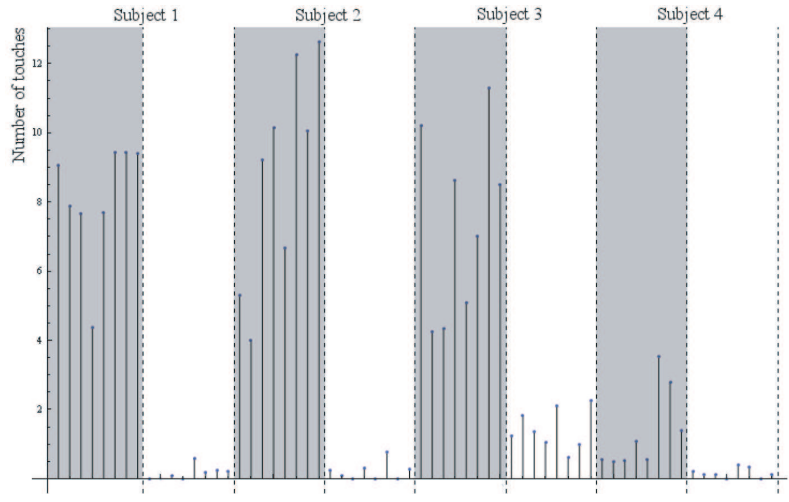

Figure 4: Number of static touches; each touch lasts between $0.5 \mathrm{~s}$ and $1.5 \mathrm{~s}$. Each pair of gray and white regions corresponds to the data of a single subject. In the gray parts the subjects were asked about the temperature of the material, in the white part about the relief. The 16 bars for each subject correspond to the 16 questions/scenes as mentioned in the text.

to keep his hand still as we were measuring hand movements. This explains the small circular movements that can be seen in his movement patterns but not in those of the other subjects.

To analyze in more detail whether the movement patterns indeed contain static touches, we determined the number of static touches for each scene and each individual subject. As subject 5 had misunderstood this task, we did not include his patterns in this analysis. We defined a "static touch" as no movement for a duration between $0.5 \mathrm{~s}$ and $1.5 \mathrm{~s}$. This upper limit was motivated by the presumed underlying physical cause of these static touches. Finger temperatures will be above the temperature of the samples (which are at room temperature). As a subject holds a finger on a single spot on a material, this spot is heated and thus the heat transfer will gradually decrease, so after a while a static touch is no longer a suitable strategy for temperature perception and the subject will move on. Longer lasting touches will have other causes, such as, for example, distraction. To define "static touch", the grid of the scenes was divided into $5 \times 5 \mathrm{~mm}$ cells; static touch is then defined as keeping one's finger within a single cell.

The result of this analysis can be seen in Figure 4. The gray areas refer to the temperature related questions, the first four bars for each subject to patterns as found in Figure 3. We can see that indeed a number of static touches occurs. We will defer the discussion whether this number is substantial or not until we have discussed the results belonging to the relief related questions.

In Figure 5 the movement patterns belonging to specific temperature comparison questions are shown. Again, the patterns seem to consist of static touches interchanged with movements to the next sample. Except for subject 5, hardly any detailed local movements can be seen (but as explained above, these are caused by a slight misunderstanding of the instructions). Even without looking at the caption, it is evident for 3 out of 5 subjects which sample had to be used as reference, as after inspecting a sample they go back to the reference. For these 3 subjects the patterns are remarkably similar.

The haptic scenes that were used in Figure 5 (scenes 6 and 7) consist of different sets of material samples (see Figure 2). Still the movement patterns found do not seem to depend on scene: the question seems to direct the movement pattern.

We also determined the number of static touches for these questions, which are shown in Figure 4, bars 5-8 of each subject. Although there is some variation from trial to trial, it can be seen that the numbers are comparable to those obtained for the other temperature related questions 1-4.

In Figure 6 the movement patterns resulting from the question "Describe the relief" can be seen for the same five subjects. Both the difference with the previous movement patterns and the consistency between the subjects is striking. All five subjects make a large number of small local movements. In many cases, the orientation of the local movements is distinct and depends on the stimulus, especially for those samples that have a clear orientation, such as samples 7, 83, 103 and 127.

These patterns suggest that static touch is not used very often when subjects have to describe the relief. As before, we determined the number of static touches, which can be seen in the white regions of Figure 4. Indeed, static touches hardly occur. To determine whether this difference between the number of static touches in the temperature related conditions and that in the relief related condi- 
1.6

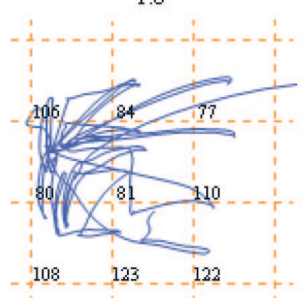

1.7

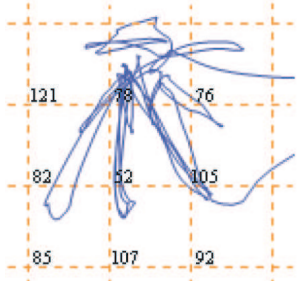

2.6

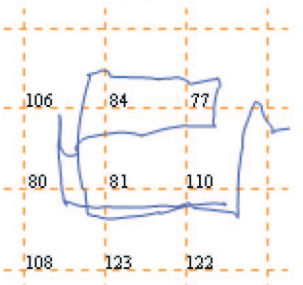

27

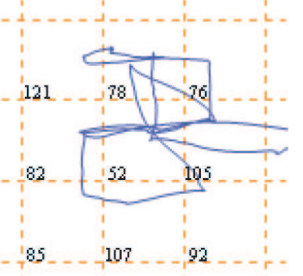

3.6



3.7

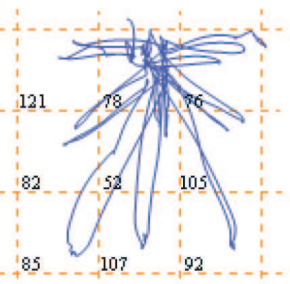

4.6

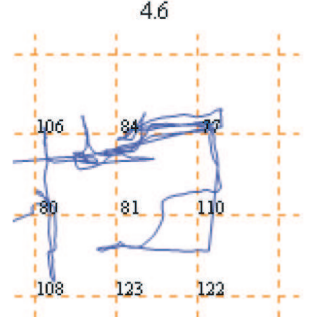

4.7

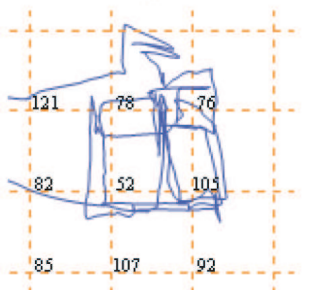

5.6

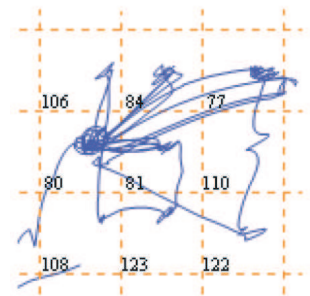

5.7

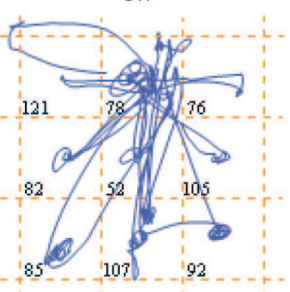

Figure 5: Movements patterns for five subjects resulting from the question "Which sample is most like the left-center (upper row) or top-center (bottom row) in terms of perceived temperature?" The first number above each scene refers to the subject, the second to the scene number. Scenes are described in Tables 1 and Figure 2.

1.9



1.10

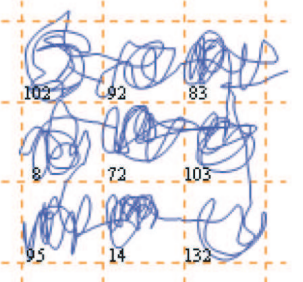

2.9

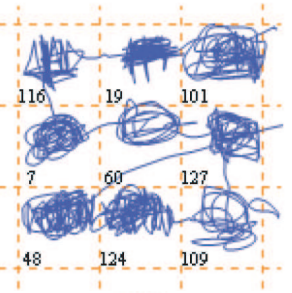

2.10

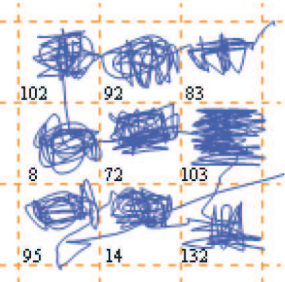

3.9

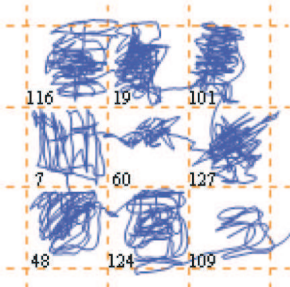

3.10

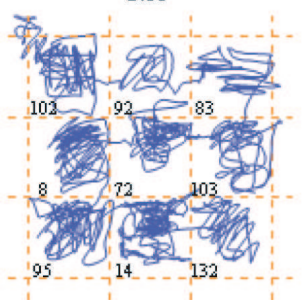

4.9

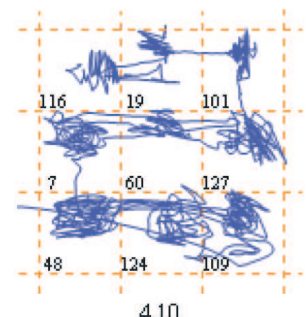

4.10

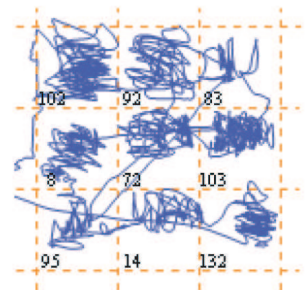

5.9

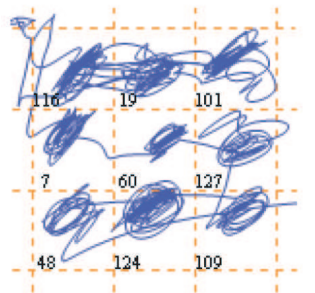

5.10

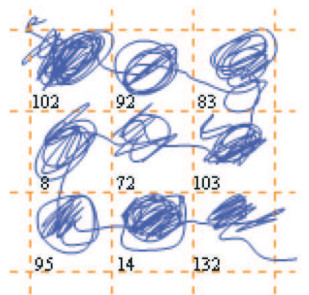

Figure 6: Example of movement patterns from five subjects resulting from the question "Describe the relief". The first number above each scene refers to the subject, the second to the scene number. Scenes are described in Tables 1 and Figure 2.

tions is statistically significant, we ran a one-sided $t$-test for each subject. For all subjects, this difference turned out to be highly significant $(p<0.0005$ for subjects 1,2 , and 3 and $p<0.05$ for subject 4$)$.

Finally, we were also interested in the question whether the local movements depended on the orientation of the relief. The scenes used in combination with questions 13-16 consisted of corrugated cardboard samples in various orientations. We observed that most movements in such cases consisted of back and forth movements along a line of a certain direction. Therefore, we decided not to distinguish between directions $\alpha$ and $\alpha+180^{\circ}$. As a consequence of this choice, the possible movement directions range from $1^{\circ}$ to $180^{\circ}$. We analyzed the movement directions of subsequent datapoints and plotted the relative number of occurence for each movement direction in steps of $1^{\circ}$.

The results of this directional analysis can be seen in Figure 7. In the first graph, the orientation of the ridges on the stimulus is $0^{\circ}$ and the movements made lie predominantly along the $0^{\circ}$ and $90^{\circ}$ directions. In the second graph of Figure 7 , the orientation of the ridges was $45^{\circ}$. It can clearly be seen, that the movement directions shifted with the stimulus orientation: peaks occur both at $45^{\circ}$ and $135^{\circ}$, although still a substantial number of the movements is made along the cardinal directions $0^{\circ}$ and $90^{\circ}$. The other graphs of this figure show similar behavior: subjects tend to direct their movements along or perpendicular to the direction of the ridges, in combination with movements along the cardinal axes.

\section{Discussion AND Conclusions}

The major research question of this paper was whether different questions about a haptic scene elicit different two-dimensional movement patterns, just like what has been found in the field of eye movement research. Although it may not be too surprising, the answer to this question is a convincing "yes". If we compare the movement patterns shown in Figures 3,5 and 6, we see a series of very distinct patterns. The questions related to temperature induce a series of static touches, followed by a smooth movement to the 



Figure 7: Direction patterns on stimuli rotated by: $0^{\circ}, 45^{\circ}, 67.5^{\circ}$, $22.5^{\circ}, 79^{\circ}$, and $169^{\circ}$, respectively. The histogram indicates the relative occurence of movements between subsequent data points in a certain direction.

next sample. If the question is formulated slightly differently and subjects have to compare perceived temperature to that of a specific sample, the movements also consist of static touches, but in between comparison samples subjects move back to the reference. Hardly any local movements can be observed in these cases. Questions related to relief yield completely different movement patterns: subjects tend to make small repetitive movements back and forth along the orientation of the stimulus or perpendicular to it, alternating with movements along the cardinal directions.

On the other hand, the movement patterns for different scenes are very similar as long as the correponding question is the same or similar. This can be observed by comparing the two rows in Figures 3,5 and 6. In the first case, the scene consists of the same materials but ordered in a different way; in the other two cases, the two scenes consist of different sets of materials. In all cases, the movement patterns made by a subject are almost identical.

Finally, the consistency of the movement patterns for the various participants is quite stunning. Similar to what was shown for eye movements by Yarbus [6], different individuals explore a haptic scene in very similar ways. For the haptic exploration of three-dimensional objects this was already shown by Lederman and Klatzky [4], who could classify a number of distinct exploratory strategies that would be used given a specific task. Surprisingly, two-dimensional hand movements have never been looked at in this particular way, whereas two-dimensional exploration of scenes is also a daily activity. The specific local movements made might have been partly expected from what we know about exploratory strategies: Estimating temperature requires static touch and texture related questions induce lateral motion. However, it is also clear that these two exploratory strategies do not suffice to describe the rich variety of movement patterns in the current, still limited, experiment on the exploration of two-dimensional haptic scenes. Our finding of this wide but consistent diversity of movement patterns opens up a way for more quantitative analyses of the patterns. In future research, we will try to classify these patterns in terms of characteristic features, such as, for example, "straightness", circularity", and "staticness".

\section{Acknowledgements}

This work was supported by the EU project no. 248587, "THE Hand Embodied".

\section{REFERENCES}

[1] W. M. Bergmann Tiest and A. M. L. Kappers. Analysis of haptic perception of materials by multidimensional scaling and physical measurements of roughness and compressibility. Acta Psychologica, 121(1):120, 2006.

[2] B. Breidegard, Y. Eriksson, K. Fellenius, K. Holmqvist, B. Jönsson, and S. Strömqvist. Enlightened: The art of finger reading. Studia Linguistica, 62(3):249-260, 2008.
[3] G. T. Buswell. How people look at pictures - A study of the psychology of perception in art. The University of Chicago Press, Chicago, Illinois, 1935.

[4] S. J. Lederman and R. L. Klatzky. Hand movements: A window into haptic object recognition. Cognitive psychology, 19(3):342-368, 1987.

[5] S. Millar. Reading by touch. Routledge, London, 1997.

[6] A. L. Yarbus. Eye movements and vision. Plenum Press, New York, 1967. 